\title{
One-Pot Preparations of Diaryliodonium Bromides from Iodoarenes and Arenes, with Sodium Perborate as the Oxidant ${ }^{\dagger}$
}

\author{
Anna Kryska and Lech Skulski*
}

Chair and Laboratory of Organic Chemistry, Faculty of Pharmacy, Medical University, Banacha 1, PL 02-097 Warsaw, Poland.

$\dagger$ This paper was presented at the Fifth Electronic Conference on Synthetic Organic Chemistry (ECSOC-5), September 1-30, 2001 (Paper A0007).

* Author to whom correspondence should be addressed; Tel/Fax: +48(22)8226843; e-mail: lskulski@farm.amwaw.edu.pl

Received: 27 September 2001; in revised form 11 October 2001 / Accepted: 12 October 2001 / Published: 31 October 2001

\begin{abstract}
This paper reports a one-pot synthesis of the title bromides from both activated and deactivated iodoarenes which are first oxidized with anhydrous $\mathrm{NaBO}_{3} \cdot \mathrm{H}_{2} \mathrm{O} / \mathrm{Ac}_{2} \mathrm{O} /$ conc. $\mathrm{H}_{2} \mathrm{SO}_{4}$ liquid mixtures, then coupled in situ with benzene and activated arenes and, finally, precipitated out with a $\mathrm{KBr}$ solution; this method is easy, cheap, safe and fairly effective.
\end{abstract}

Keywords: diaryliodonium bromides, iodoarenes, arenes, sodium perborate as oxidant

\section{Introduction}

Symmetric and unsymmetric diaryliodonium salts, $\operatorname{Ar}_{2} I^{+} X^{-}$and $\operatorname{Ar}\left(\operatorname{Ar}^{\prime}\right) I^{+} X^{-}$, represent an important class of aromatic iodine(III) derivatives. They are used in organic synthesis mostly as arylating reagents for a large variety of organic and inorganic nucleophiles. Some of them display a biological activity or photochemical properties, hence they are widely used as efficient catalysts for radiation initiated polymerisation. Generally, their reactivity is less pronounced than that of other hypervalent 
iodine(III) compounds. They are solid compounds, mostly stable towards heat (particularly those with substantially nonnucleophilic counterions), oxygen, and humidity; they are mildly light-sensitive and should be stored in the dark, without refrigeration [1].

Indeed, there a multitude of different published methods for their preparation are available [1], and the relevant literature is still expanding nowadays with dislosures of some novel and possibly better methods [2]. In our opinion, some of these methods are rather costly and/or too complicated to be easily adopted in "ordinary" organic laboratories - but it is still possible and desirable to devise some new preparative procedures, which would be easy, cheap, safe, and effective. We hope that a novel method reported in this paper may fulfil, at least in part, these expectations.

\section{Results and Discussion}

In 1995 we reported [3] a one-pot ("short-cut") synthesis of sparingly soluble diaryliodonium bromides (and iodides) from various iodoarenes, ArI, previously oxidized with anhydrous $\mathrm{CrO}_{3} / \mathrm{AcOH} / \mathrm{Ac}_{2} \mathrm{O} /$ conc. $\mathrm{H}_{2} \mathrm{SO}_{4}$ systems to form soluble iodine(III) intermediates, $\mathrm{ArISO}_{4}$ or $\mathrm{ArI}\left(\mathrm{OSO}_{3} \mathrm{H}\right)_{2}$ (not isolated), then coupled in situ with benzene and activated arenes/heteroarenes (Ar'H) and, finally, precipitated out with excess aqueous $\mathrm{KBr}$ (or $\mathrm{KI}$ ) solutions. The title bromides were obtained in $20-88 \%$ crude yields (for more information see Ref. [3]). This method was easier and shorter then many earlier methods [1], hence it was later often used in our laboratory [4-6], and elsewhere [7].

We also reported $[3,5]$ the very simple and effective oxidative metatheses in the crude iodonium bromides, iodides and chlorides into the corresponding pure tetrafluoroborates, tosylates, triflates, trifluoroacetates, etc.; such diaryliodonium salts with substantially nonnucleophilic counterions are the best arylating reagents [1]. However, there are some important, inherent limitations in the application of our short-cut method [3]. Only iodobenzene, haloiodobenzenes, and iodoarenes substituted with electron-withdrawing groups may effectively be applied to form the final iodonium bromides (or iodides). 4-Iodotoluene coupled oxidatively with benzene gave $4-\mathrm{MeC}_{6} \mathrm{H}_{4}(\mathrm{Ph}) \mathrm{I}^{+} \mathrm{Br}^{-}$in only $20 \%$ crude yield, whereas iodoanisoles and iodoacetanilides were oxidatively cleaved by $\mathrm{CrO}_{3}$ in acidic medium, hence their oxidative coupling with activated arenes was not possible. Thus, we have been looking for some novel and effective one-pot method, which would also be applicable for iodoarenes substituted with electron-donating groups. In fact, we have increasingly been interested in the mild and selective oxidative properties of sodium perborate (which is purchased either as "monohydrate" or "tetrahydrate" at a low price) widely used in chemical laboratories or in industry. It may be considered as a "dry carrier" of the hazardous and unstable hydrogen peroxide, is easy to handle, safe, and stable at room temperature [8]. Therefore, we have decided to use $\mathrm{NaBO}_{3} \cdot \mathrm{H}_{2} \mathrm{O}$ as the oxidant of choice, because it is more soluble in acetic anhydride than $\mathrm{NaBO}_{3} \cdot 4 \mathrm{H}_{2} \mathrm{O}$. We know from our earlier work that water, due to its high hydration power, diminishes the oxidizing activity of inorganic (and organic) oxidants.

McKillop and Kemp [9] oxidized thirteen ArI (also those substituted with electron-donating groups!) to the corresponding $\mathrm{ArI}(\mathrm{OAc})_{2}$ with $\mathrm{NaBO}_{3} \cdot 4 \mathrm{H}_{2} \mathrm{O}$ (used in $900 \%$ excess) in a very large volume of glacial acetic acid within $4-8$ hours, at $40-50^{\circ} \mathrm{C}$. After recrystallizations, they obtained the 
purified products in 66-80\% yields. When we used acetic anhydride as the solvent of choice and $\mathrm{NaBO}_{3} \cdot \mathrm{H}_{2} \mathrm{O}$ as the oxidant, which allowed to lower considerably an excess of the oxidant applied to only $200 \%$ as well as the volume of the solvent applied, we obtained five $4-\mathrm{XC}_{6} \mathrm{H}_{4} \mathrm{I}(\mathrm{OAc})_{2}(\mathrm{X}=\mathrm{H}, \mathrm{F}$, $\mathrm{Br}, \mathrm{Cl}, \mathrm{MeO})$ as well as $2-\mathrm{MeOC}_{6} \mathrm{H}_{4} \mathrm{I}(\mathrm{OAc})_{2}$ in 60-87\% crude yields; after repeated recrystallizations, we obtained the purified products [10]. This preliminary work opened up a new way to develop the title one-pot method. We have also observed that a preliminary period of stirring $\mathrm{NaBO}_{3} \cdot \mathrm{H}_{2} \mathrm{O}$ with acetic anhydride at $30{ }^{\circ} \mathrm{C}$ (preferably within $90 \mathrm{~min}$ ) enhances considerably the oxidizing activity of this solution. Recently, Indian chemists [11] have observed the generation of peracetic acid on aging of $\mathrm{NaBO}_{3} \cdot 4 \mathrm{H}_{2} \mathrm{O}$ solutions in glacial acetic acid, confirmed by chemical tests.

The essence of our novel method is given in the three equations below; for all preparative details see Experimental.

(1) $\mathrm{ArI}+\mathrm{NaBO}_{3} \cdot \mathrm{H}_{2} \mathrm{O}+2 \mathrm{Ac}_{2} \mathrm{O}+2 \mathrm{H}_{2} \mathrm{SO}_{4} \rightarrow \mathrm{ArI}\left(\mathrm{OSO}_{3} \mathrm{H}\right)_{2}+\left[\mathrm{NaBO}_{2}\right]+4 \mathrm{AcOH}$

(2) $\mathrm{ArI}\left(\mathrm{OSO}_{3} \mathrm{H}\right)_{2}$ (not isolated) $+\mathrm{Ar}^{\prime} \mathrm{H}\left(400 \%\right.$ excess) $\rightarrow \mathrm{Ar}\left(\mathrm{Ar}^{\prime}\right) \mathrm{I}^{+} \mathrm{HSO}_{4}{ }^{-}+\mathrm{H}_{2} \mathrm{SO}_{4}$

(3) $\mathrm{Ar}\left(\mathrm{Ar}^{\prime}\right) \mathrm{I}^{+} \mathrm{HSO}_{4}^{-}$(not isolated $)+$aq. $\mathrm{KBr}$ (in excess) $\rightarrow \downarrow \mathrm{Ar}\left(\mathrm{Ar}^{\prime}\right) \mathrm{I}^{+} \mathrm{Br}^{-}+\mathrm{KHSO}_{4}$

Small samples of the crude bromides were quickly [3] recrystallized from $\mathrm{MeOH}$ to give the pure analytical samples. Their melting points agreed well with the literature data [3, 12], and their structures were confirmed by correct elemental analyses (Table 1) as well as ${ }^{1} \mathrm{H}-\mathrm{NMR}$ spectra (not shown here); the spectra (in DMSO- $\mathrm{d}_{6} / \mathrm{DDS}$ ) displayed the sharp singlets located at ca. 2.20, 2.45, 2.75, and 4.20 ppm, characteristic of the $\mathrm{COCH}_{3}, 4-\mathrm{CH}_{3} \mathrm{Ar}$, 2- and 2,6-CH $\mathbf{C H}_{3} \mathrm{Ar}$, or 4-OCH $\mathrm{H}_{3}$ groups, respectively, as well as composite multiplets characteristic of the aromatic protons (the thoroughful integrations disclosed the total numbers of aromatic protons related to the aforesaid three-proton singlets); cf. our former papers $[3,5]$.

\section{Conclusions}

In this paper we present a novel one-pot method for easy and effective preparation of a number of diaryliodonium bromides (Table 1). They were produced from both activated and deactivated iodoarenes and 2-iodothiophene, which next were oxidatively coupled with activated arenes, in appropriate anhydrous $\mathrm{NaBO}_{3} \cdot \mathrm{H}_{2} \mathrm{O} / \mathrm{Ac}_{2} \mathrm{O} /$ conc. $\mathrm{H}_{2} \mathrm{SO}_{4}$ mixtures. Next, the iodonium bromides were precipitated out by the addition of excess $\mathrm{KBr}$ solution to the resulting final reaction mixtures. After their collection, washing, and air-drying, the final products were obtained in $23-98 \%$ crude yields; only small samples were purified and analysed. Sodium perborate monohydrate used as the oxidant is cheap, safe and effective; no toxic residues were left after the reactions - in a strong contrast to our former method [3].

\section{Experimental}

\section{General}

All iodoarenes, arenes, solvents as well as inorganic reagents were pure commercial products. The melting points of the purified diaryloiodonium bromides (Table 1) are uncorrected, and were measured 
as follows. Since they depend on the duration of heating, after an approximate mp was taken in an open capillary tube, a new sample was introduced about $10{ }^{\circ} \mathrm{C}$ below this point, and the temperature was then raised at a rate of $4-5{ }^{\circ} \mathrm{C}$ per minute. ${ }^{1} \mathrm{H}-\mathrm{NMR}$ solution spectra (not shown here) were recorded with a Tesla (100 MHz) spectrometer. Microanalyses were carried out at the Institute of Organic Chemistry, the Polish Academy of Sciences, Warsaw.

\section{One-Pot Preparations of Diaryliodonium Bromides}

$\mathrm{NaBO}_{3} \cdot \mathrm{H}_{2} \mathrm{O}(37.5 \mathrm{mmol})$ was suspended in $\mathrm{Ac}_{2} \mathrm{O}(17 \mathrm{~mL})$, and this mixture was stirred at $30{ }^{\circ} \mathrm{C}$ for $90 \mathrm{~min}$. An appropriate iodoarene $(12.5 \mathrm{mmol})$ was then added, the mixture was stirred at $40{ }^{\circ} \mathrm{C}$ for $1.5 \mathrm{~h}$, and at that point benzene or an appropriate activated arene $(5 \times 12.5 \mathrm{mmol})$ was added, and the mixture was cooled to $5-10{ }^{\circ} \mathrm{C}$. Conc. $(98 \%) \mathrm{H}_{2} \mathrm{SO}_{4}(2.5 \mathrm{~mL})$ was slowly added dropwise, with stirring, while keeping the temperature below $15{ }^{\circ} \mathrm{C}$. Next, the mixtures were stirred, at appropriate temperature (10-30 ${ }^{\circ} \mathrm{C}$ ) for a given time ( $4-6 \mathrm{~h}$, but with benzene $48 \mathrm{~h}$; see Table 1 for details). The final reaction mixtures were poured into cold water $(150 \mathrm{~mL})$ and stirred for $0.5-1 \mathrm{~h}$. The unreacted organic substrates were extracted with $\mathrm{Et}_{2} \mathrm{O}(2 \times 20 \mathrm{~mL})$, and the ethereal extracts were discarded. The aqueous layers, if dark-coloured, were decolourized with charcoal while boiling. To the cooled filtrates, excess aq. $\mathrm{KBr}(6 \mathrm{~g} \mathrm{KBr}$ in $30 \mathrm{~mL}$ water) was added with stirring. After $1-2 \mathrm{~h}$, the precipitates were collected, washed well with water, acetone, and $\mathrm{Et}_{2} \mathrm{O}$, air-dried, and weighed to give the iodonium bromides in $23-98 \%$ crude yields (Table 1). Note. When the iodoarenes and/or arenes used were solid compounds, which rendered the stirring more difficult, then an additional amount of $\mathrm{Ac}_{2} \mathrm{O}$ (5-10 mL) was added to the reaction mixtures, prior to the addition of conc. $\mathrm{H}_{2} \mathrm{SO}_{4}$.

Table 1. Preparative Details, Crude Yields, and Melting Points of the Purified Diaryliodonium Bromides 1-24 as well as their Elemental Analyses

\begin{tabular}{|c|c|c|c|c|c|}
\hline $\begin{array}{l}\text { Substrates } \\
\text { ArI, Ar'H }\end{array}$ & $\begin{array}{l}\text { Product } \\
\mathbf{A r}\left(\mathbf{A} \mathbf{r}^{\prime}\right) \mathbf{I}^{+} \mathbf{B r}^{-}\end{array}$ & $\begin{array}{l}\text { Time }[\mathrm{h}] / \\
\text { Temp. }\left[{ }^{\circ} \mathrm{C}\right]^{\mathrm{a}}\end{array}$ & \begin{tabular}{|c|} 
CrudeYield \\
{$[\%]$} \\
\end{tabular} & $\mathrm{Mp}\left[{ }^{0} \mathrm{C}\right]$ & $\begin{array}{l}\left.\text { Analyses [ } \begin{array}{ll}\% & \text { I}\end{array}\right] \\
\text { Calcd/Found }\end{array}$ \\
\hline $\mathrm{PhI}, \mathrm{PhH}$ & Diphenyliodonium bromide (1) & $48 / 30$ & 51 & $246-247$ & $35.15 / 35.3$ \\
\hline PhI, PhMe & $\begin{array}{l}\text { 4-Methyldiphenyliodonium bromide } \\
\text { (2) }\end{array}$ & $5 / 15$ & 65 & $199-200$ & $33.83 / 33.6$ \\
\hline $\begin{array}{l}\text { PhI, } \\
\text { Mesitylene }\end{array}$ & $\begin{array}{l}\text { 2,4,6-Trimethyldiphenyliodonium } \\
\text { bromide (3) }\end{array}$ & $5 / 15$ & 82 & $166-167$ & $31.48 / 31.6$ \\
\hline PhI, PhOMe & $\begin{array}{l}\text { 4-Methoxydiphenyliodonium bromide } \\
\text { (4) }\end{array}$ & $5 / 15$ & 91 & $211-212$ & $32.45 / 32.4$ \\
\hline PhI, PhNHAc & $\begin{array}{l}\text { 4-Acetylaminodiphenyliodonium } \\
\text { bromide (5) }\end{array}$ & $5 / 25$ & 30 & $202-203$ & $30.35 / 30.5$ \\
\hline PhI, Thiophene & $\begin{array}{l}\text { Phenyl(2-thienyl)iodonium bromide } \\
\text { (6) }\end{array}$ & $5 / 15$ & 55 & $206-207$ & $34.57 / 34.9$ \\
\hline
\end{tabular}




\begin{tabular}{|c|c|c|c|c|c|}
\hline $\begin{array}{l}\text { 2-I-Thiophene, } \\
\text { 3- } \mathrm{MeC}_{6} \mathrm{H}_{4} \mathrm{OMe}\end{array}$ & $\begin{array}{l}\text { 4-Methoxy-2-methylphenyl(2- } \\
\text { thienyl)iodonium bromide (7) }\end{array}$ & $6 / 15$ & 23 & $154-155$ & $32.19 / 32.4$ \\
\hline $\begin{array}{l}\text { 2-I-Thiophene, } \\
\text { Thiophene }\end{array}$ & 2,2'-Dithienyliodonium bromide (8) & $6 / 15$ & 30 & $183-184$ & $34.01 / 34.4$ \\
\hline $\begin{array}{l}\text { 2- } \mathrm{MeC}_{6} \mathrm{H}_{4} \mathrm{I} \\
\mathrm{PhOMe}\end{array}$ & $\begin{array}{l}\text { 4-Methoxy-2'-methyldiphenyl- } \\
\text { iodonium bromide (9) }\end{array}$ & $6 / 15$ & 50 & $200-201$ & $31.22 / 31.5$ \\
\hline $\begin{array}{l}\text { 3- } \mathrm{MeC}_{6} \mathrm{H}_{4} \mathrm{I}, \\
\mathrm{PhOMe}\end{array}$ & $\begin{array}{l}\text { 4-Methoxy-3'-methyldiphenyl- } \\
\text { iodonium bromide (10) }\end{array}$ & $6 / 15$ & 55 & $171-172$ & $31.22 / 31.5$ \\
\hline $\begin{array}{l}\text { 4-MeC }{ }_{6} \mathrm{H}_{4} \mathrm{I} \\
\mathrm{PhOMe}\end{array}$ & $\begin{array}{l}\text { 4-Methoxy-4'-methyldiphenyl- } \\
\text { iodonium bromide (11) }\end{array}$ & $6 / 15$ & 70 & 194-195 & $31.22 / 31.4$ \\
\hline $\begin{array}{l}\text { 2-MeOC }{ }_{6} \mathrm{H}_{4} \mathrm{I} \\
\mathrm{PhOMe}\end{array}$ & $\begin{array}{l}\text { 2,4'-Dimethoxydiphenyliodonium } \\
\text { bromide (12) }\end{array}$ & $5 / 15$ & 70 & $223-224$ & $30.04 / 29.9$ \\
\hline $\begin{array}{l}\text { 3-MeOC }{ }_{6} \mathrm{H}_{4} \mathrm{I} \\
\mathrm{PhOMe}\end{array}$ & $\begin{array}{l}\text { 3,4'-Dimethoxydiphenyliodonium } \\
\text { bromide (13) }\end{array}$ & $5 / 15$ & 48 & $178-179$ & $30.04 / 30.0$ \\
\hline $\begin{array}{l}\text { 4- } \mathrm{MeOC}_{6} \mathrm{H}_{4} \mathrm{I} \\
\mathrm{PhOMe}\end{array}$ & $\begin{array}{l}\text { 4,4'-Dimethoxydiphenyliodonium } \\
\text { bromide (14) }\end{array}$ & $5 / 15$ & 50 & $210-211$ & $30.04 / 30.1$ \\
\hline $\begin{array}{l}\text { 2-AcNHC}{ }_{6} \mathrm{H}_{4} \mathrm{I}, \\
\mathrm{PhOMe}\end{array}$ & $\begin{array}{l}\text { 2-Acetylamino-4'-methoxydiphenyl- } \\
\text { iodonium bromide (15) }\end{array}$ & $5 / 15$ & 50 & 193-194 & $28.32 / 27.9$ \\
\hline $\begin{array}{l}\text { 3-AcNHC }{ }_{6} \mathrm{H}_{4} \mathrm{I} \\
\mathrm{PhOMe}\end{array}$ & $\begin{array}{l}\text { 3-Acetylamino-4'-methoxydiphenyl- } \\
\text { iodonium bromide (16) }\end{array}$ & $5 / 15$ & 45 & $198-200$ & $28.32 / 27.9$ \\
\hline $\begin{array}{l}\text { 4-AcNHC}{ }_{6} \mathrm{H}_{4} \mathrm{I} \\
\mathrm{PhOMe}\end{array}$ & $\begin{array}{l}\text { 4-Acetylamino-4'-methoxydiphenyl- } \\
\text { iodonium bromide (17) }\end{array}$ & $5 / 10$ & 40 & 206-207 & $28.32 / 28.6$ \\
\hline $\begin{array}{l}\text { 4- } \mathrm{ClC}_{6} \mathrm{H}_{4} \mathrm{I}, \\
\text { 1,3-Xylene }\end{array}$ & $\begin{array}{l}\text { 4-Chloro-2',4'-dimethyldiphenyl- } \\
\text { iodonium bromide (18) }\end{array}$ & $5 / 15$ & 55 & $185-186$ & $29.96 / 30.1$ \\
\hline $\begin{array}{l}\text { 4- } \mathrm{ClC}_{6} \mathrm{H}_{4} \mathrm{I} \\
\text { Mesitylene }\end{array}$ & $\begin{array}{l}\text { 4-Chloro-2',4',6'-trimethyl- } \\
\text { diphenyliodonium bromide (19) }\end{array}$ & $5 / 15$ & 25 & 201-202 & $29.00 / 29.4$ \\
\hline $\begin{array}{l}4-\mathrm{ClC}_{6} \mathrm{H}_{4} \mathrm{I} \\
\mathrm{PhOMe}\end{array}$ & $\begin{array}{l}\text { 4-Chloro-4'-methoxydiphenyl- } \\
\text { iodonium bromide (20) }\end{array}$ & $6 / 15$ & 87 & $205-206$ & $29.83 / 30.1$ \\
\hline $\begin{array}{l}\text { 4- } \mathrm{AcC}_{6} \mathrm{H}_{4} \mathrm{I} \\
\mathrm{PhOMe}\end{array}$ & $\begin{array}{l}\text { 4-Acetyl-4'-methoxydiphenyl- } \\
\text { iodonium bromide (21) }\end{array}$ & $5 / 15$ & 96 & $167-168$ & $29.21 / 29.0$ \\
\hline $\begin{array}{l}2-\mathrm{O}_{2} \mathrm{NC}_{6} \mathrm{H}_{4} \mathrm{I} \\
\mathrm{PhOMe}\end{array}$ & $\begin{array}{l}\text { 4-Methoxy-2'-nitrodiphenyliodonium } \\
\text { bromide (22) }\end{array}$ & $6 / 15$ & 96 & $185-186$ & $29.10 / 29.2$ \\
\hline $\begin{array}{l}3-\mathrm{O}_{2} \mathrm{NC}_{6} \mathrm{H}_{4} \mathrm{I} \\
\mathrm{PhOMe}\end{array}$ & $\begin{array}{l}\text { 4-Methoxy-3'-nitrodiphenyliodonium } \\
\text { bromide (23) }\end{array}$ & $4 / 15$ & 98 & $175-176$ & $29.10 / 28.9$ \\
\hline $\begin{array}{l}4-\mathrm{O}_{2} \mathrm{NC}_{6} \mathrm{H}_{4} \mathrm{I} \\
\mathrm{PhOMe}\end{array}$ & $\begin{array}{l}\text { 4-Methoxy-4'-nitrodiphenyliodonium } \\
\text { bromide (24) }\end{array}$ & $4 / 15$ & 55 & $157-158$ & $29.10 / 29.0$ \\
\hline
\end{tabular}

a Time and temperature of the acidic coupling reaction between a prior oxidized ArI and an appropriate Ar'H. 


\section{References and Notes}

1. (a) Olah, G. A. Halonium Ions. Wiley: New York, 1975; (b) Koser, G. F. In The Chemistry of Functional Groups. Suppl. D Patai, S.; Rappoport, Z., Eds.; Wiley-Interscience: Chichester, 1983, Chapter 25; (c) Varvoglis, A. The Organic Chemistry of Polycoordinated Iodine. VCH: Weinheim, 1992; (d) Koser, G. F. In The Chemistry of Halides, Pseudo-Halides and Azides, Suppl. D2 Patai, S.; Rappoport, Z., Eds.; Wiley-Interscience: Chichester, 1995, Chapter 21; (e) Stang, P. J.; Zhdankin, V. V. Chem. Rev. 1996, 96, 1123; (f) Varvoglis, A. Hypervalent Iodine in Organic Synthesis Academic Press: San Diego, 1997.

2. (a) Ochiai, M.; Toyonari, M.; Sueda, T.; Kitagawa, Y. Tetrahedron. Lett. 1996, 37, 8421; (b) Shah, A.; Pike, V. W.; Widdowson, D. A. J. Chem. Soc., Perkin Trans. 1, 1997, 2463; (c) Roh, K. R.; Kim, J. Y.; Kim, Y. H. Chem. Lett. 1998, 1095; (d) Ochiai, M.; Yutaka, Y.; Takayama, N.; Takaoka, Y.; Shiro, M. J. Am. Chem. Soc. 1999, 121, 9233; (e) Kitamura, T.; Wakimoto, I.; Nakamura, T.; Fujiwara, Y. Org. Lett. 1999, 253; (f) Kitamura, T.; Yamane, M.; Inoue, K.; Todaka, M.; Fukatsu, N.; Meng, Z.; Fujiwara, Y. J. Am. Chem. Soc. 1999, 121, 11674; (g) Pike, V. W.; Butt, F.; Shah, A.; Widdowson, D. A. J. Chem. Soc., Perkin Trans. 1 1999, 245; (h) Kitamura, T.; Meng, Z.; Fujiwara, Y. Tetrahedron. Lett. 2000, 41, 6611; (i) Carrol, M. A.; Pike, V. M.; Widdowson, D. A. Tetrahedron Lett. 2000, 41, 5393; (j) Grushin, V. V. Chem. Soc. Rev. 2000, 29, 315; (k) Peacock, M. J.; Pletcher, D. Tetrahedron Lett. 2000, 41, 8995.

3. Kazmierczak, P.; Skulski, L. Synthesis, 1995, 1027.

4. Farhan, A. N.; Skulski, L. Pol. J. Chem. (a) 1995, 69, 1663; (b) 1996, 70, 211; (c) 1997, 71, 1236.

5. Kazmierczak, P.; Skulski, L. Bull. Chem. Soc. Jpn. 1997, 70, 219.

6. Skulski, L.; Wroczynski, P. Bull. Pol. Acad. Sci., Chem. 1999, 47, 231.

7. Zhou, T.; Chen, Z. - C. J. Chem. Res., Synop. 2001, 153, see p 154.

8. (a) Muzzart, J. Synthesis 1995, 1325; (b) McKillop, A.; Sanderson, W. R. Tetrahedron 1995, 51, 6145; (c) McKillop, A.; Sanderson, W. R. J. Chem. Soc., Perkin Trans. 1 2000, 471.

9. McKillop, A.; Kemp, D. Tetrahedron 1989, 45, 3299.

10. Skulski, L. Molecules, 2000, 5, 1331, pp 1353-1354 and 1361. Avail. at URL: http://www.mdpi.org/molecules/papers/51201331.pdf

11. Karunakaran, C.; Kamalan, R. Eur. J. Org. Chem. 2000, 3261.

12. (a) Beringer, F. M.; Gindler, E. M. Iodine Abstr. Rev. 1956, 3, 1; Drexler, M.;Gindler, E. M.; Lumpkin, C. C. J. Am. Chem. Soc. 1953, 75, 2705; (c) Beringer, F. M.; Bachofner, H. E.; Falk, R. A.; Leff, M. J. Am. Chem. Soc. 1958, 80, 4279; (d) Beringer, F. M.; Karniol, M.; Lillien, I.; Masullo, G.; Mausner, M.; Sommer, E. J. Am. Chem. Soc. 1959, 81, 342.

Sample availability: Samples of crude compounds 1-24 are available from the authors. Samples of pure compounds 4, 9-11, 14-16 are available from MDPI.

(C) 2001 by MDPI (http://www.mdpi.org). Reproduction is permitted for noncommercial purposes. 